\title{
AM ANFANG WAR DIE TECHNIK \\ Zu Bernard Stieglers zeit-technischer Verspätung des Menschen
}

von MARIE-LUISE ANGERER

Bernard Stiegler, Die Logik der Sorge. Verlust der Aufklärung durch Technik und Medien, Frankfurt/M. (Suhrkamp) 2008, übers. v. Susanne Baghestani

Ders., Von der Biopolitik zur Psychomacht, Frankfurt/M. (Suhrkamp) 2009, übers. v. Susanne Baghestani

Ders., Technik und Zeit. Der Febler des Epimetheus, Berlin, Zürich (diaphanes) 2009, übers. v. Gabriele Ricke und Ronald Vouillé

Ders., Denken bis an die Grenzen der Maschine, hg. und mit einem Vorwort von Erich Hörl, Berlin, Zürich (diaphanes) 2009, übers. v. Ksymena Wojtyczka

Ders., Hypermaterialität und Psychomacht, hg. und mit einem Vorwort von Erich Hörl, Berlin, Zürich (diaphanes) 2oro, übers. v. Ksymena Wojtyczka

Jacques Derrida, Bernard Stiegler, Echographien. Fernsebgespräche, hg. von Peter Engelmann, Wien (Passagen Verlag) 2006, übers. v. Horst Brühmann

Erich Hörl (Hg.), Die technologische Bedingung: Beiträge zur Beschreibung der technischen Welt, Berlin (Suhrkamp) 20 I I

\section{Technik und Zeit}

Im Jahr 1993 führte Jacques Derrida mit Bernard Stiegler in seinem Haus in Ris-Orangis ein langes Gespräch über damals hochaktuelle medientechnologische Entwicklungen: Dies waren angesichts der von CNN übertragenen Bombenabwürfe über Bagdad die Direktübertragung und die Produktion der digitalen Bilder.

«In Echtzeit zielt das Fernsehen auf ein Plusquampräsens, das alle Selektions- und Manipulationsmöglichkeiten bei der Produktion der Bilder hinter einer vermeintlich objektiven Aktualität verschwinden läßt.» ${ }^{1}$ Der Philosoph der Différance - des ursprünglichen Aufschubs als absenter Voraussetzung für jede Positivität - ringt in diesem Gespräch mit der Frage, was es bedeuten könnte, "seine Zeit zu denken», und kommt zum Schluss: "Sie ist ein Artefakt». ${ }^{2}$ Diese Zeit, die Zeit der Wiederholung, der Dekonstruktion, der Politik und der Medien, bildet in Stieglers Arbeit den Fokus - sie wird bei ihm zu einer ursprünglichen und technischen Zeit: «Der Mensch ist die Technik, das heißt die Zeit.» ${ }^{3}$

Technik und Zeit (so der Titel des Hauptwerks von Stiegler in Abwandlung von Heideggers Sein und Zeit) bildet jedoch nicht nur für Bernard Stiegler den Rahmen einer technikphilosophischen und medientheoretischen Abhandlung über die Gegenwart, sondern diese beiden Pole - Technik und Zeit - bilden den Hintergrund für zahlreiche medientheoretische Versuche, aktuelle medientechnische Entwicklungen in den Griff zu bekommen.

(Medien-)Techniken werden dabei als konstituierende, disziplinierende, formierende Macht gefasst, die die Beziehung von Natur-Kultur-Technik-Gesellschaft-Individuum bestimmen. Autoren wie Canguilhem, Simondon und Kittler, Hayles, Thacker, Hansen, Latour, Haraway und Stiegler können hierzu u. a. genannt werden.

Soeben ist der Band von Erich Hörl Zur technischen Bedingung mit Beiträgen einiger dieser Autoren erschienen. Stiegler wird dort als Wortführer einer Organologie vorgestellt, wie sie von Kapp über Canguilhem und Simondon vorgelegt bzw. angedacht worden ist. Hierbei spielt Stieglers Begriff des "pharmakologischen Wissens» eine zentrale Rolle, wie er in Von der Biopolitik zur Psychomacht eingeführt wird. Dort, als Kritik an Foucault und dessen blin den Flecken einer pharmakologischen Herausforderung 
gegenüber, formuliert Stiegler Elemente eines Programms der Sorge um sich und die anderen. Wie lassen sich, so seine Frage, die guten Techniken des Selbst von jenem «pharmakon» der Programmindustrien und des Marketing unterscheiden? Wie kann die Materialität der technischen Objekte mit ihrer Kraft zur Individuierung und gleichzeitigen Desindividuation als "Produktionsmaschine» ${ }^{4}$ heute begriffen werden, wo die Foucault'sche Biomacht doch nicht mehr zur Erklärung ausreicht, weil sie noch das alte Europa im Blick hat sowie auf eine Praxis abzielt, die heute aufgrund viraler Marketingstrategien überholt ist. Aus einer staatlichen Biomacht ist längst eine «Psychomacht des Marktes» ${ }^{5}$ geworden. Wie also sind, so Stiegler in seinem Aufsatz «Allgemeine Organologie und positive Pharmakologie», ${ }^{6}$ die drei organologischen Ebenen - die psychosomatischen Organe des psychischen Individuums, die technischen und künstlichen Organe des technischen Individuums sowie die gesellschaftlichen Organe wie Institutionen und Organisationen aller Art - in ihren transduktiven Beziehungen zu erforschen? Eine Organologie, die die psychischen, technischen und kollektiven Individuen als konkrete «Dispositive der Individuation» begreift, muss im Sinne Stieglers also über die Psychomächte der Märkte geschrieben werden, um die Verschränkung globaler Finanzmärkte mit der Wiedererstehung der Slums rund um Paris und der inflationären Zunahme depressiver Erkrankungen analysieren zu können und um hieraus ein Technik- und Medienverständnis abzuleiten, das dem materiell-geistigen Komplex der Individuation heute gerecht wird.

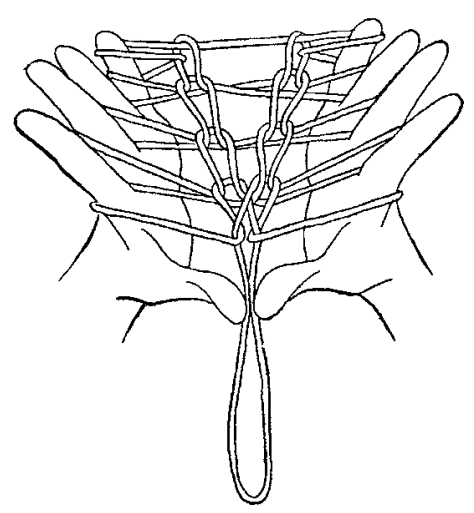

Der «ursprüngliche Mensch», schreibt Stiegler in Anlehnung an Rousseau, Leroi-Gourhan, Nietzsche u.a. war immobil und sprachlos («Die Sprache gehört bereits zur Prothese» ${ }^{7}$ ). Im Augenblick seines Falls (aus dieser Ursprünglichkeit heraus) wird der Mensch das Sein verfehlen - ein «ursprünglicher Fehl», so Stiegler (bei Lacan war von «manque à l'être» die Rede, ebenfalls mit Blick auf Heidegger) wird ihn fortan begleiten. Dieses Nichthaben des Seins, geschuldet der Vergesslichkeit des Epimetheus, der alle Gaben verteilte, um dann entdecken zu müssen, dass für den Menschen nichts mehr übrig geblieben war, wird zur Lebensbedingung des Menschen, er ist fortan damit beschäftigt, diesen «Ursprungsfehler aus(zu)gleichen, indem er sich mit Prothesen, mit Instrumenten versieht.» ${ }^{8}$ Das heißt, der Mensch entspringt einer ursprünglichen Vergessenheit, die ihn nun zwingt, sich von sich zu trennen, seine ursprüngliche Immobilität und Stummheit aufzugeben und auf die Objekte da draußen zurückzugreifen.

\section{Bewusstsein und Zeitobjekte}

Von der Höhlenmalerei über die Keilschrift zur Fotografie, weiter zu Film und Fernsehen bis zu Computer, Internet und Smartphone ist die Geschichte der Menschheit eine der Zeitobjekte, die das verhandeln, was den Menschen, sein Gedächtnis und damit seine Geschichte ausmacht: Vergangenheit, Gegenwart und Zukunft. Heute jedoch, so Stiegler in Denken bis an die Grenzen der Maschine, erleben wir eine "Ekstase der Zeit», ${ }^{9}$ die wesentlich der Industrialisierung des 20. und 21. Jahrhunderts geschuldet ist. Der Mensch als epiphylogenetisches Wesen wird von seinen Mnemotechniken bestimmt, doch diese leiten gegenwärtig Zeitigungsprozesse und damit Individuationsprozesse ein, die Stiegler als «Industrialisierungsprozess des Gedächtnisses» ${ }^{10}$ beschreibt, eine "politische und industrielle Ökonomie, die auf der industriellen Ausbeutung von Bewusstseinszeiten beruht»." Die Konsumentenkörper als Egomassen bilden die globalen Absatzmärkte einer Industrie, die die Bewusstseine dieser Körper degradieren und zerstören, vergleichbar der Ausrottung von Tierarten und der Zerstörung natürlicher Ressourcen. Um zu begreifen, was dieser industrielle Zugriff bedeutet, der in den Augen Stieglers eine mental kranke, infantilisierte Gesellschaft produziert, die an einer allgemeinen Störung der Aufmerksamkeit leidet und zu struktureller Verantwortungslosigkeit tendiert, ${ }^{12}$ müsse man sich Husserls Theorie der Zeitobjekte vor Augen führen. Dort hatte Husserl die aufbewahrte Vergangenheit im Hier und Jetzt analysiert und 


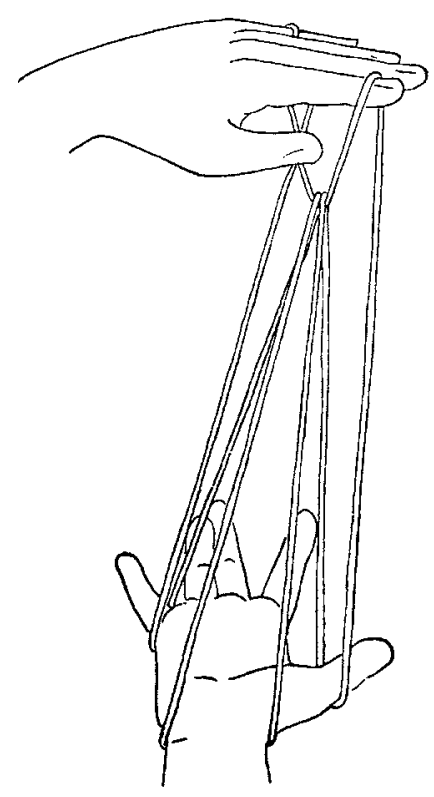

dieses Zurückbehalten oder Speichern des z. B. vorausgegangenen Tons in der gegenwärtigen Wahrnehmung als «primäre Retention» bestimmt. Von dieser unterschied er die «sekundäre Retention", die der Vergangenheit angehört, also Erinnerung oder Fantasie ist. Während Husserl diese beiden Retentionen radikal trennte, stehen sie bei Stiegler nicht nur in Kombination miteinander, sondern werden darüber hinaus durch die «tertiäre Retention» ergänzt, die den Umstand benennt, dass durch die technische Speicherung etwas jederzeit abrufbar ist, doch jede Wiederholung unterschiedliche Wirkungen hervorruft. Diese Möglichkeit sei heute, so Stiegler, in eine neue Epoche getreten, die auch das Ende des Okzidents bedeuten könnte. Tertiäre Retentionen sind orthothetisch, phonogrammatisch, kinematografisch oder alphabetisch - auf jeden Fall sind es Objekte, technische Objekte, die - und hier nun kommt die Frage des Okzidents ins Spiel - die Epoche der Geschichte, des Rechts, der Philosophie und der Wissenschaft einleiten. ${ }^{13}$ Stiegler sieht den Okzident durch den Prozess der Industrialisierung und Kommerzialisierung des Gedächtnisses jedoch in eine große Krise geraten, die zugleich eine Katastrophe der Geistesgeschichte ist, die er als «allgemeinen Entfaltungsprozess der menschlichen, individuellen und kollektiven Zeitlichkeit» versteht, «die aus gespenstischen «Wiederkünften〉 und Wiederholungen besteht [...] Angesichts des Unbehagens in der Welt [...], das mit der Liquidierung jahrtausendalter psychischer und kollektiver Individuationsprozesse einhergeht, [...] sind ultrareaktionäre Versuchungen der Xenophobie, diverse Fanatismen [...] und alle möglichen Formen von Ressentiments die unvermeidliche Folge.» ${ }^{14}$ Nicht länger mehr ließen sich Gesellschaften in linke oder rechte Politiken unterscheiden, sondern nur mehr in Sorgetragende und Fahrlässige unterteilen. ${ }^{15}$ Die Medien haben dabei die Aufgabe übernommen, jeden Widerstand auszuschalten, um eine größtmöglichste Durchlässigkeit und Akzeptanz der Produkte und Programme (der Bewusstseinsindustrie) zu gewährleisten.

\section{Psychomacht, Biopolitik, Hypermaterialität}

Der Psychoanalyse Freuds hat, so Stiegler, eine Theorie der tertiären Retention als «Theorie der Materialisierung der verräumlichten Zeit und [...] der Hypermaterie» ${ }^{16}$ gefehlt. Diese Hypermaterialität hat sich nach Stiegler mit der Quantenmechanik entwickelt und bezeichnet «einen Komplex aus Energie und Information, in dem Materie nicht mehr von ihrer Form unterschieden werden kann». ${ }^{17}$ Hypermateriell ist jener Prozess, «in dem die Information - die als Form erscheint - in Wirklichkeit eine Folge von Zuständen der Materie ist, die durch Hardware, durch Apparate, durch techno-logische Vorrichtungen und Dispositive erzeugt wird». ${ }^{18}$ Genau jedoch in jenem Moment, wo die Unterscheidung von Form und Materie sinnlos geworden ist, kann die Psychomacht nach Stiegler ihre ungehemmte Wirkungsmacht entfalten. Die von Stiegler als Triebkapitalismus bezeichnete kapitalistische Entwicklungsstufe verändert das Sublimierungsvermögen, die Wissenschaft sowie die geistigen Aktivitäten insgesamt. Wir befinden uns, so Stiegler, bereits in einem «sehr gefährlichen Entsublimierungsprozess». ${ }^{19}$ Während die Wissenschaft im 20. Jahrhundert einen Prozess des Technowissenschaftlich-Werdens durchlaufen hätte, wodurch sie zu einer Art Science Fiction geworden ist, zu einer Produzentin von Trugbildern, hat die Industrie der Wunschökonomie gleichzeitig ihr Begehren ausgetrieben. Es ist interessant, die zentrale Rolle des Wunsches sich in Erinnerung zu rufen, wie sie Deleuze und Guattari einmal formuliert haben (als Kritik an der Psychoanalyse und am Kapitalismus), um bei Stiegler auf dessen sehr veränderte Bedeutung und Rolle zu treffen. Nach Stiegler ist jede Gesellschaft auf libidinösen Besetzungen aufgebaut, der Kapitalismus als solcher funktioniert selbst nur über derartige Besetzungen. Doch die Entwicklungen im vorigen Jahrhundert gehen weit über das hinaus, was die 
alte Marx'sche Lehre als Warenfetischismus bezeichnet hat - das wichtigste Ziel sei nämlich längst nicht mehr die Reproduktion der Arbeitskraft, sondern die Bindung des Konsumenten. ${ }^{20}$ Hauptantriebskraft dieser Entwicklung sind die Massenmedien, die nicht nur die traditionellen Familienstrukturen zerstören können, sondern auch diejenigen der Demokratien. Stieglers Fazit über die Frankfurter Schule: Ihre Mitglieder waren unfähig zu erkennen, dass das Marx'sche Denken für eine Konsumanalyse nicht ausreicht. ${ }^{21}$

Die Mitglieder der Frankfurter Schule haben die Massenmedien der Verdummung der Menschen bezichtigt, haben ihnen vorgeworfen, aus mündigen Bürgern abhängige und kritiklose couch potatoes zu machen, um sie umso freiwilliger der Berieselung durch Werbung und Politik auszusetzen. Hiervon unterscheidet sich die Argumentation von Stiegler nicht wirklich. Die Differenz betrifft wesentlich jedoch zwei Punkte - zum einen sind für Stiegler nicht mehr die klassischen Massenmedien wie Radio und Fernsehen die zentralen Instanzen, sondern primär die neuen Propagandamächte Marketing und Public-Relation, und zum anderen gibt es in der Arbeit von Stiegler keinen Begriff der Ideologie mehr. Haben die Massenmedien in den Augen der Frankfurter Schule noch auf den Überbau und damit auf das Bewusstsein sowie Unbewusste der Menschen gezielt, ist die Unterscheidung zwischen Sein und Bewusstsein der hypermateriellen Kondition (s.o.) gewichen. Deshalb kann Erich Hörl im Vorwort zum Band Hypermaterialität auch als zentrales Anliegen von Stiegler formulieren, «die Begriffe zu erfinden, mit denen sich die heutige libidoökonomische Situation bestimmen lässt». ${ }^{22}$ Denn der Wunsch, so die These Stieglers, werde von tiefen Umwälzungen des kapitalistischen Gesamtgebarens seit dem auslaufenden 19. Jahrhundert einer ständigen Umschrift unterzogen und drohe heute in seiner «industriellen Bearbeitung" zur Erschöpfung gebracht zu werden. Das Einlassen der technischen Objekte (als tertiäre Retentionen) in diese Wunschökonomie muss gerade heute, so kann ein Fazit aus den Arbeiten Stieglers lauten - nach Psychoanalyse, nach Foucault und Deleuze \& Guattari -, das Denken von Technik in ihrer Zeit wiederum bestimmen.

\begin{tabular}{|c|c|}
\hline 1 Derrida, Stiegler, Echographien, 2. & 11 Ebd. \\
\hline 2 Ebd., 13. & 12 Stiegler, Die Logik der Sorge. \\
\hline 3 Stiegler, Technik und Zeit, 157. & 13 Stiegler, Denken bis an die \\
\hline 4 Stiegler, Von der Biopolitik zur & Grenzen der Maschine, 77. \\
\hline Psychomacht, 50. & 14 Ebd., 78. \\
\hline 5 Ebd., 53. & 15 Stiegler, Von der Biopolitik zur \\
\hline 6 Stiegler, Allgemeine Organolo- & Psychomacht. \\
\hline gie und positive Pharmakologie & 16 Stiegler, Hypermaterialität und \\
\hline (Theorie und praxis), in: Hörl & Psychomacht, 119. \\
\hline (Hg.), Die technologische Bedingung, & 17 Ebd., 105. \\
\hline $110-146$ & 18 Ebd. \\
\hline 7 Stiegler, Technik und Zeit, 157f. & 19 Ebd., 119. \\
\hline 8 Ebd., 156. & 20 Ebd., 50. \\
\hline 9 Stiegler, Denken bis an die & 21 Ebd., 51. \\
\hline Grenzen der Maschine, 69. & 22 Ebd., 17. \\
\hline
\end{tabular}

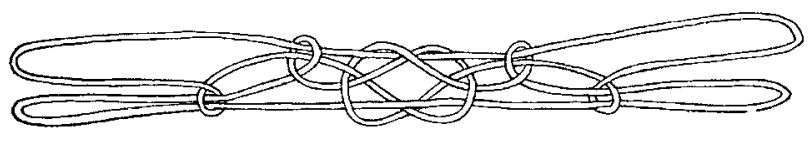




\section{STRING FIGURES}

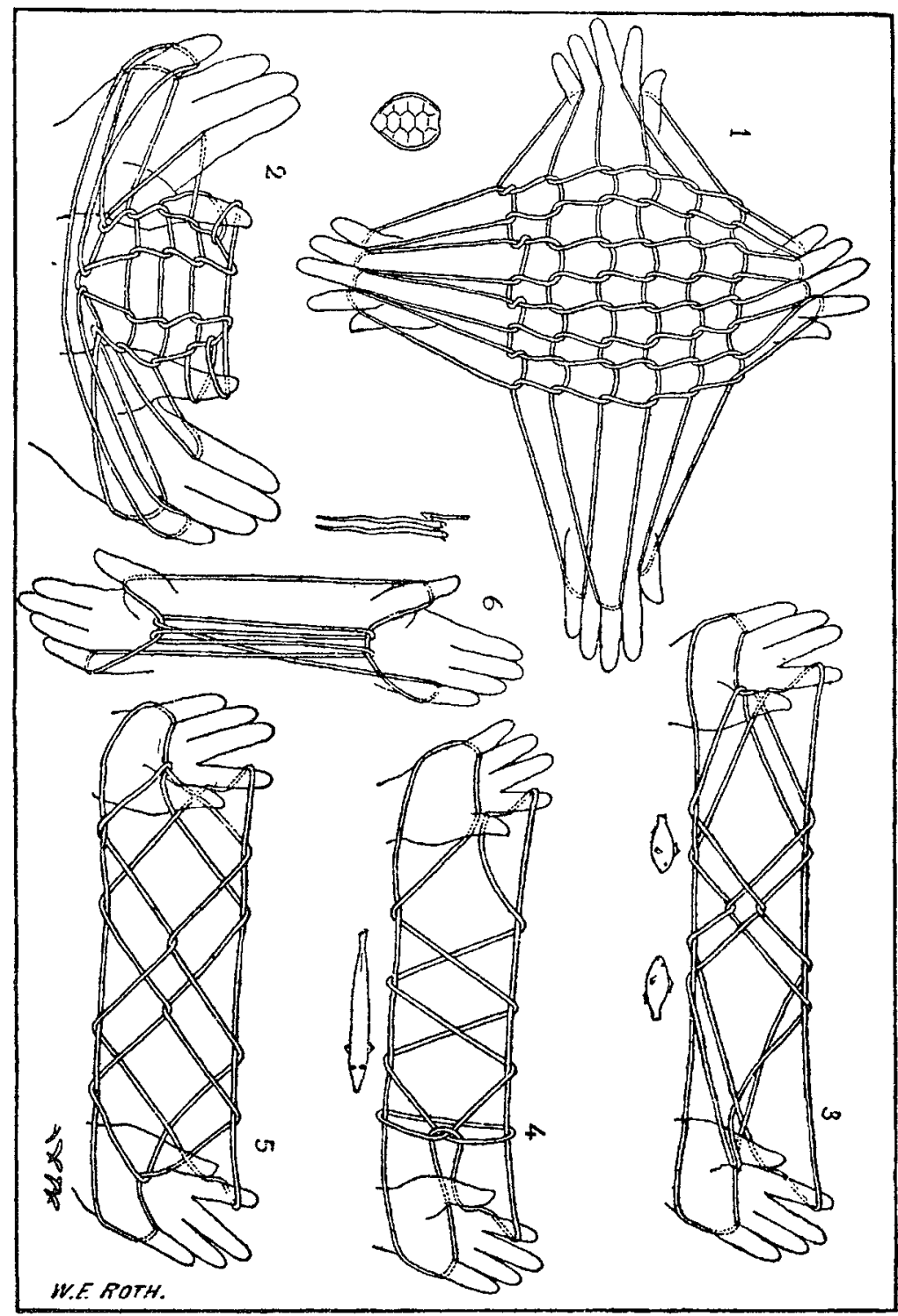

Roth's Plate VII.-Animals: Reptileg and Fish

1. Tortoise: the scutum. (Middle) Palmer River,

2. Turtle: the scutum. Pennefather River.

3. Two fish. (Lower) Tully River.

4. Fish. Atherton.
5. Mullet skimming along the water. Cape Grafton.

6. Eels carried on a hooked stick (a common method of carrying fish). Cape Bedford. 